\title{
Reflexiones sobre el curso de extensión y derechos humanos «Afrodescendencia: conceptos en torno a una noción activa»
}

\author{
Fernanda Olivar Rodríguez, Lourdes Martínez Betervide, ${ }^{2}$ \\ Victoria Pereira Beltran, ${ }^{3}$ Julio E. Pereyra Silva ${ }^{4}$
}

Recibido: 28/03/2021; Aceptado: 4/06/2021

DOI: https://doi.org/10.37125/ISR.7.1.4

\section{Resumen}

En este artículo se expone la experiencia docente a partir de las dos ediciones del curso de extensión con enfoque en derechos humanos «Afrodescendencia: conceptos en torno a una noción activa». En 2019 el curso giró en torno a afrodescendencia y derechos humanos y en el segundo, ya de forma virtual, en la racialización de la pandemia. Proponemos compartir una experiencia de docencia afrocentrada, y a partir de ella, reflexionar acerca de la educación desde un abordaje étnico-racial, con perspectiva de género y generaciones y de carácter interdisciplinario. Representación social de la afrodiáspora, situación de las mujeres y jóvenes afrodescendientes y educación antirracista, son las tres líneas de acción que desarrollamos desde el Grupo de Investigación Colectivo de Estudios Afrolatinoamericanos, responsables del Cursos de Extensión con Enfoque en Derechos Humanos 2019 y 2020. De estas experiencias recogemos aprendizajes e interrogantes acerca de la extensión universitaria transversalizada por la dimensión étnico racial, la aparente omisión de atención a la población afrouruguaya en la historia de la extensión universitaria de nuestra universidad, la responsabilidad de la Udelar de visibilizar las problemáticas y actuar

1 Facultad de Humanidades y Ciencias de la Educación (FHCE), Universidad de la República (Udelar); Departamento de Medicina Familiar y Comunitaria (Facultad de Medicina, Udelar); grupo de trabajo «Crisis civilizatoria, reconfiguraciones del racismo, movimientos afrolatinos» (Clacso 2019-2022); Colectivo de Estudios Afrolatinoamericanos (csic, Udelar). fernandaolivar87@gmail.com

2 Facultad de Derecho, Udelar; grupo de trabajo Afrodescendencias y Propuestas Contrahegemónicas (Clacso 2019-2022); Colectivo de Estudios Afrolatinoamericanos (csic, Udelar). martinezbetervide@gmail.com

3 FHCE, Udelar; Jóvenes Afro; Colectivo de Estudios Afrolatinoamericanos (CSIC, Udelar). vitopereira1621@gmail.com

4 Instituto Escuela Nacional de Bellas Artes (IENBA), Udelar; Facultad Latinoamericana de Ciencias Sociales (Argentina); Tecnicatura en Tecnologías de la Imagen Fotográfica (IENBA, Udelar); Asociación de Investigadores Afrolatinoamericanos y del Caribe; Colectivo de Estudios Afrolatinoamericanos (csic, Udelar). jempereyra@gmail.com 
desde la integralidad, de cara a identificar, estudiar y transformar el racismo y sus consecuencias.

Palabras clave: afrodescendencia, Uruguay, educación antirracista, extensión, interdisciplina.

\section{Extensión y derechos humanos como campos de acción antirracista}

La tradición extensionista uruguaya, hija del proceso reformista cordobés de 1918 se ha desarrollado desde la máxima de dar "prioridad a los sectores más postergados». A la luz de esta consigna han transcurrido más de cien años en los que la Universidad de la República (Udelar) se ha embarcado en la búsqueda de prácticas pedagógicas horizontales, procesos de enseñanza aprendizaje democráticos «fundamentado en la necesidad de superar el modelo de enseñanza universitaria tradicional profesionalista, retórico y fragmentador» (Tommasino, Cano, Castro, Santos y Stevenazzi, 2017, p. 1). Producto de una tradición educativa que da por sentada y natural la lejanía de lo producido desde la universidad respecto a lo experimentado desde algunos sectores de la sociedad, la extensión universitaria ha volcado cada uno de sus esfuerzos en la superación de este paradigma desde acciones que rompan las paredes materiales y simbólicas del aula. Desde las carreras tradicionales pasando por las «ciencias duras» hasta las humanidades y ciencias sociales y las artes, todas han consolidado al día de hoy experiencias de extensión universitaria, cada una con desafíos y resistencias disciplinarias propias. Es insoslayable la conexión de las humanidades y ciencias sociales con las prácticas en territorio o el trabajo con la comunidad, en tanto a pesar de sus vestiduras academicistas se reconoce en ellas «lo social» como un valor consustancial.

Dar «prioridad a los sectores más postergados» invita a entender la educación como un derecho, y por ende evidencia un posicionamiento político, ético y académico que llama a interpelar a un sistema educativo que, aunque universal, laico y gratuito

... produce resultados fuertemente vinculados a los niveles socioeconómicos de los que provienen sus estudiantes [...] resulta claro que el sistema educativo uruguayo, en toda su extensión, funciona reproduciendo y naturalizando las desigualdades sociales que existen en la propia sociedad de la que forma parte (Martinis, 2016, p. 18).

Y al pensar en desigualdades y en el foco de atención de nuestro trabajo, se hace necesario recordar que en el Uruguay contemporáneo la población afrodescendiente se encuentra ubicada en su mayoría en el quintil más pobre de nuestra sociedad. La sobrerrepresentación estadística en los niveles de pobreza da cuenta, entre otros elementos, de cómo son afectados en un alto grado por el analfabetismo y la deserción estudiantil temprana.5 Según datos del último Censo Nacional de Población, de

5 Datos del Censo Nacional de Población realizado en 2011 (Instituto Nacional de Estadística, 2011). 
cada diez estudiantes afro que culminan primaria, tres completan el ciclo básico de la educación media y solo uno alcanza el bachillerato. Si la educación pública uruguaya sostiene los valores de universal, laica y gratuita, ¿a qué responden estas cifras?, ¿Qué posibilidades hay de encontrar en la educación una herramienta de emancipación y libertad?

Con estos datos a la vista, a la hora de diseñar un curso que una la extensión con los derechos humanos en relación con la afrodescendencia, nuestra propuesta se centró en la comprensión de los procesos que este término lleva en sí, las tensiones, tanto políticas y teóricas, que lo rodean. En este entendido es que nos centramos en el carácter «activo» de la afrodescendencia, en tanto creemos en la necesidad de abordarla no como una noción estática y unidimensional, sino desde un abordaje que remita a los diversos procesos nacionales e internacionales que desembocaron en su formación. Procesos que conllevan en sí tensiones, avances y marchas atrás, así como una larga cadena de aprendizajes colectivos, protagonizados por diferentes actores en el ámbito de lo que se denomina la diáspora africana.

Para ello proponemos pensar el aula como un espacio de encuentro, en donde confluyen quienes tienen dudas sobre el tema, junto a aquellos que pueden dar voz a diferentes aspectos relevantes de este carácter activo antes mencionado.

La selección de estas voces, en atención a la concepción de educación afrocentrada (categoría que explicitaremos en otro apartado), fue pensada en función de entender que no era posible encontrarlas en el ámbito académico. Cuatro mujeres afrouruguayas, militantes, con responsabilidades en el ámbito público en diversas áreas y con trayectorias vitales y educativas, en las que incluso para algunas de ellas entra la experiencia universitaria, que tenían como centro la temática afro. Fueron invitadas a exponer en la primera edición Beatriz Ramírez, Verónica Villagra, Elizabeth Suárez y Vicenta Camusso. Para la segunda edición, donde el tema propuesto giraba en torno de la pandemia de covid-19 tomamos el mismo criterio de traer estas voces que no encontraban eco en el ámbito de la enseñanza universitaria e invitamos a Miguel Pereira, Tania Ramírez, Verónica Villagra y el colectivo Somos Negras y Qué.

En la primera edición los puntos tratados se relacionan a la experiencia de vida en función de una agenda vinculada a los temas de derechos humanos que de forma más evidente impactan en los afrodescendientes:

- Derechos políticos de los afrodescendientes y los procesos relacionados a su reivindicación

- Derechos de las mujeres afrodescendientes y formas de organización en la diáspora

- Seguridad social y derechos humanos 
- Instrumentos de protección de derechos de los afrodescendientes en los espacios de gobierno en el Uruguay. el caso de la Secretaría de Equidad Étnico Racial y de Poblaciones Migrantes en la intendencia de Montevideo

En la segunda edición, donde el tema giraba en torno a la pandemia de covid-19 los temas tratados fueron:

- $\quad$ Salud de los afrodescendientes

- Acciones desde los espacios gubernamentales con perspectiva étnico-racial.

- La pandemia como un fenómeno feminizado y racializado

- Situación de la pandemia en el marco de la diáspora africana, con énfasis en Latinoamérica

En el programa de las dos ediciones de este curso afirmamos que los procesos socio-políticos que rodean la noción de afrodescendencia ha tenido un intenso devenir $y$, en el medio nacional, estos se desarrollado tanto desde los procesos liderados por la sociedad civil organizada hasta la incorporación de la temática en diversos formatos en la estructura del Estado. Esto nos muestra que la condición de activa, tal como el título del curso propone, se hace evidente al ponerse en relación con otros, tanto espacios como individuos, y en su propia condición de activa los modifica allí en donde se pone en juego.

En el contexto en el que estamos insertos, apelar a esta condición activa le imprime a la afrodescendencia la complejidad necesaria para lograr establecer una multiplicidad de frentes insoslayables, cada uno de los cuales requieren múltiples acciones, las que van desde dar a conocer los procesos históricos por lo que se llegó al surgimiento del término, las diferentes injusticias que este intenta reparar, hasta la revisión del impacto que esta nueva forma de posicionamiento político de la población afro ha tenido en nuestra sociedad. Proceso que da cuenta tanto de las resistencias generadas hasta de su inclusión favorable en diversos espacios.

Entendemos que en tanto es un concepto nuevo en términos históricos, encuentra fricciones en los modos estructurantes del vínculo social a la vez que necesita de estrategias que garanticen una cabal comprensión y completa inserción, en tanto estas formas de la estructura social cuentan a su favor con una naturalización del vínculo que opaca la comprensión de las formas de injusticia que conlleva. Así, los espacios de enseñanza, en sus diversos niveles y formatos, si bien ofrecen la posibilidad de desplegar estas estrategias, también forman parte de este entramado de los mencionados modos estructurantes.

Como principal estrategia este curso propone traer al espacio del aula la voz de diversos actores, sociales, académicos, políticos para escucharlos en primera persona. Desde los grupos organizados en acciones solidarias, hasta aspectos vinculados a la 
salud y por supuesto, la educación, aspectos que, si antes eran complejos, en el proceso pandémico se hacen urgentes.

\section{Ennegrecer la academia: cuerpos afro en la institución}

La población estudiantil de grado de la Udelar concentra un 89,8 \% de estudiantes activos en Montevideo y la matrícula en el interior alcanza un 10,2\%. Los datos existentes ${ }^{6}$ respecto a la conformación del universo de estudiantes en esta casa de estudios reafirma la baja participación de la población afro $(3,9 \%)$ a nivel de los registros oficiales del Estado en el espacio educativo. Y esta ausencia cuantitativa es el correlato de una realidad cualitativa ya que en la práctica la Udelar parece ser una universidad «blanca». Para entender mejor esta afirmación basta también mirar la composición de los cuerpos docentes o de los estudiantes de posgrado.

Este equipo docente compuesto por cuatro profesionales provenientes del campo de las artes, educación, derecho y antropología, todas disciplinas en apariencia alejadas unas de otras o sutilmente próximas por pertenecer a la denominada área social y artística por la Universidad de la República. Conforman desde 2018 el Colectivo de Estudios Afrolatinoamericanos, registrado en la Comisión Sectorial de Investigación Científica (CSIC) y centra su actividad en el estudio de las condiciones de vida de la población afrodiaspórica en América Latina, con énfasis en el estudio de los efectos del racismo en la educación y las representaciones sociales.

Tres mujeres y un hombre afro, que trabajan sobre, desde y por la afrodescendencia, persiguiendo el objetivo de romper con el ciclo estructural de violencia epistémica, convocan a seis mujeres y un hombre afro en una clara intención política de hacer del cuerpo docente un acto disruptivo bajo la convicción de que «no habrá justicia social sin justicia cognitiva», como plantea Boaventura de Sousa Santos (2009). Cuerpos que no forman parte del cuerpo docente universitario, que en su mayoría tampoco han sido invitados a formar parte de lo universitario ni relevantes en tanto productores de pensamiento. En términos performáticos configuran

... una acción estratégica de visibilidad y reconocimiento: cuatro compañeras que conjugan en ellas la historia del movimiento de mujeres afrodescendientes del Uruguay, del movimiento feminista, de la incidencia política en nuestra agenda de derechos (nacional e internacional), referentes de distintos espacios de y en la construcción de políticas públicas (Martínez, Olivar, Pereira y Pereyra, 2020, p. 170).

Subyace a este programa de formación el intento consciente de lograr formas de diálogo entre las epistemologías universitarias tradicionales y las afro epistemologías (García, 2010, 2018) con el objetivo de potenciar el feedback entre teoría y práctica con una clara perspectiva étnico racial. La propuesta de un modelo pedagógico afrocentrado es a la vez una apuesta política en la búsqueda de acciones académicas

6 Los datos utilizados en este trabajo responden al perfil de los estudiantes de grado de la Universidad de la República 2018 Form-A Relevamiento continuo. Dirección General de Planeamiento (DGP), Udelar. 
desde donde trazar pensamientos, teorías y tradiciones con la reconstrucción de una genealogía epistémica africana y afrodiaspórica históricamente excluida de la oferta formativa, por ser considerada consciente o inconscientemente inferior pues emergen desde el sur global y son producidas desde personas oprimidas dentro de este contexto geopolítico.

Como plantea el afrovenezolano Jesús Chucho García (2018):

Occidente intentó imponernos, y aún continúan haciéndolo, dos tipos de pedagogía. La primera fue la pedagogía de la sumisión, donde la iglesia jugó un papel fundamental a través de las prácticas religiosas forzadas [...]. La otra pedagogía, fue la de la reproducción, entendiéndola como el proceso de asimilación del conocimiento despreciativo del otro hacia nosotros, reproducido en el sistema educativo formal con un currículum bien estructurado y castrante sobre nuestros conocimientos ancestrales [...] las prácticas pedagógicas del cimarronaje están en lucha abierta, y permanentemente contra las prácticas de la pedagogía de la reproducción (p. 68).

Consideramos a estas «formas otras» de saberes y conocimientos, relevantes para ser transmitidas en tanto ofrecen miradas que dinamizan y tensionan el corpus tradicional legitimado, habilitan la circulación —el cimarronaje $-^{7}$ de las producciones de los y las sujetas afrodescendientes desde la contemporaneidad de sus existencias así como, de la vigencia de los planteos de aquellos teóricos considerados clásicos como es el caso de los caribeños Aimé Césaire, Frantz Fanon, Senghor Leopold Sedar, de las intelectuales estadounidenses bell hooks, ${ }^{8}$ Kimberlé Williams Crenshaw, Angela Davis, de las intelectuales latinoamericanas del talante de Sueli Carneiro, Lélia Gonzales, Beatriz Nascimento, Luisa Bairros, Ochy Curiel, Mara Viveros, y por último, pero no menos importante, intelectuales africanos como el antropólogo congolés-brasilero Kabenguele Munanga, el escritor keniano Ngugi wa Thiongo y Achille Mbembe filósofo, teórico político e intelectual camerunés o la socióloga nigeriana experta en género Oyèrónkẹ Oyèwùmí. El carácter epistemológico de una enseñanza afrocentrada reside justamente en aceptar el reto de elaborar un programa con contenidos de autores y autoras que «por ser autores negros, las industrias editoriales han boicoteado la traducción de estos autores y las grandes universidades los han ignorado y excluido de sus currículos» (Grosfoguel, 2018, p. 11).

La corpo-política del conocimiento ha sido para este equipo un elemento central a considerar, no solo por los desafíos que implica llenar de contenido el programa, sino además, y por sobre todo, por comprender que el espacio de extensión universitaria abre una posibilidad única de acercar al espacio formal e institucional de la

7 Práctica pedagógica sustentada en una acción política consciente de lograr la circulación de producciones de autores, autoras, intelectuales afrodescendientes con la finalidad de contrarrestar la exclusión que el currículo educativo formal eurocentrista impone. Busca fomentar los conocimientos producidos desde África y la diáspora africana en una práctica indisciplinada que fomente innovaciones en las formas de enseñanza-aprendizaje.

8 Este seudónimo lo toma la autora del nombre de su bisabuela materna y prefiere no utilizar las mayúsculas por considerarlo una forma más de manifestación patriarcal. Su verdadero nombre era Gloria Jean Watkins, quien fue una intelectual, escritora y activista afroestadounidense. 
educación a aquella población cuyo vínculo se ha visto coartado a lo largo de su trayecto por múltiples formas de violencia simbólica y material intersectadas y amplificadas por la discriminación racial. Por este camino, en 2020, al ver imposibilitados los encuentros presenciales apostamos a la virtualidad a pesar de entender, que, para las poblaciones más vulnerables esta no es una opción que democratice el acceso y permanencia educativa, sino al contrario suele desembocar en un factor más hacia la consolidación de la brecha educativa.

La relación de las poblaciones afrodiaspóricas con la educación se fragilizó aún más, en tanto ya estaba afectada por diferentes formas de la discriminación racial y la desigualdad estructural histórica y ahora se suma los efectos del distanciamiento social. El encuentro para el desarrollo de futuras formas de ciudadanía, no puede reproducirse desde modelos que tienden a una experiencia educativa individual y mediada por diferentes dispositivos. Se pierde el espacio-tiempo educativo y sus características únicas. Pensar el futuro requiere de estar con y para el otro en una empresa en común. Escatimar esta experiencia es sin dudas sabotear el futuro (Pereyra, 2020, p. 3).

Y si de futuro hablamos, en medio de la incertidumbre actual signada por la pandemia, proyectamos la posibilidad de continuar ocupando este espacio de formación abierto a todo público, con énfasis en las relaciones dialógicas y horizontales entre los participantes desde la integración de las funciones universitarias. Continuamos la apuesta a este espacio fomento de formas asociativas, colectivas, plurales de pensar el mundo actual, sus embates y desafíos, apostamos a construir una educación que abone pensamiento crítico en sus participantes e interlocutores, que no dibuje fronteras epistémicas entre el adentro y el afuera y que se nutra ineludiblemente de los movimientos sociales, las demandas, las acciones y las prácticas de nuestro tiempo y espacio, poniendo así en constante refutación o comprobación todo aquello que se nos pretende aprehender acríticamente. Una educación atenta al devenir de las relaciones sociales, que aporte elementos para la identificación, prevención y acción contra cualquier tipo de vulneración de derechos hacia los y las conciudadanas, y bregar así por justicia social. Apostamos a la transformación social a través de una educación antirracista.

\section{Formación en las contradicciones de la vida: raza-etnia/clase/género}

El diseño del currículo del sistema educativo formal responde desde sus orígenes al desarrollo de conocimientos instrumentales en tanto, conforme el avance del capitalismo los sistemas educativos han moldeado los contenidos de cara a las demandas tecnológicas, técnicas y económicas de las sociedades industriales globalizadas y, sobre todo, al mercado laboral. La instrumentalización de la educación tiene una segunda función que responde a la construcción de estrategias de blanqueamiento (Munanga, 2008; Silva Bento, 2002) del proyecto de conformación del Estado nación. El ocultamiento de las intenciones subyacentes de la colonización europea en 
el mundo y, muy especialmente, en América Latina y el Caribe, así como en Asia y África, sostenida hasta los años setenta del siglo xx, la producción de conocimiento desde la visión de la historia desde el punto de vista «de los vencedores» y entendida de manera unívoca, unidimensional y unilinealmente, nos ubica en tanto personas racializadas en la «zona del no ser» (Fanon, 2008).

En un país como Uruguay en el cual el racismo es entendido y narrado como una experiencia no explícita y desarrollada de forma silenciosa casi como si esto fuera un valor, sus efectos pueden comenzar a explicarse de modo explícito desde un análisis de factores sociales y económicos. En tanto esencializa a las poblaciones e impregna de argumentos naturales las desigualdades sociales, podemos encontrar el racismo uruguayo cristalizado en la idea, arraigada con fuerza en el imaginario social, que dice que las personas negras son pobres, los pobres normalmente no llegan a la universidad, la universidad no es lugar para las personas negras, porque las personas negras son pobres.

Esta triada raza/etnia-género-clase es la que sostenemos que ha estado totalmente ausente de la visión de nuestra sociedad y por tanto de nuestra universidad, en tanto, en el Uruguay la población afrodescendiente es el 10,5\% de la población, pero representa en $16 \%$ de la población bajo la línea de pobreza, o casi un $4 \%$ de la población universitaria. Es decir, está sobrerrepresentada en la población «más postergada»o casi ausente de los espacios que pueden conducirla a una movilidad social ascendente. La ausencia física de la población afrodescendiente del ámbito universitario es, por tanto, a las claras producto de un racismo institucional, desarrollado bajo la naturalización de las desigualdades sociales y los efectos del racismo estructural. Bajo este modelo profesionalista, productivista y tecnocrático de educación, los roles de educador y educando han cristalizado en el imaginario social quienes pueden/deben o es esperable que ocupen esos roles. Por tanto, es a través de la educación que se producen y reproducen preconceptos referentes al racismo y sexismo, así como otros tantos temas que no integran el currículo educativo formal, sino hasta hace pocas décadas.

Como plantea el profesor Dr. Munanga (2020) tomando a Nelson Mandela:

... ningún infante nace racista, ni odiando a otro infante por causa del color de su piel, sino que lo hacen porque en la educación fueron socializadas en el rechazo de la diversidad y sus diferencias de la riqueza de la humanidad. En ese sentido, solo la propia educación es capaz de deconstruir los monstruos que creó y construir nuevos individuos que valoricen y convivan con la diferencia. La cuestión es saber qué tipo de educación ofrecer.

Bajo este entendido y atendiendo a los requerimientos formales del curso, el equipo definió articular y tejer vínculos con actores no universitarios desde dos aspectos.

El primero, sostenido en que el curso está dirigido a egresados, estudiantes y público en general interesado en la temática propuesta, sin requisitos excluyentes para 
inscribirse y que habilita el acceso de la población afrodescendiente a las actividades de formación, en especial a las que atañen a su situación, un aspecto sobre el que trabajamos con especial énfasis al tomar en cuenta su baja participación en la vida universitaria y considerar una medida necesaria la apertura de cursos como el que proponemos más allá del nivel de formación alcanzado. Pues, consideramos el espacio universitario, en tanto lugar de encuentro e intercambio de ideas, es propicio para trabajar en en la lucha antirracista y reflexionar sobre las posibilidades de mejora de las condiciones de vida de la población afrodescendiente. La segunda forma de vínculo es invitar a participar en el curso a actores relevantes de la comunidad afrodescendiente en las temáticas planteadas. Para ello se prevén encuentros en los cuales se convida a referentes sociales y políticos del movimiento afrouruguayo.

La filósofa afrobrasilera Djamila Ribeiro, en su trabajo O que é o lugar da fala señala que las experiencias de esos grupos, los afrodescendientes en nuestro caso, localizados socialmente de forma jerarquizada y no humanizada hace que las producciones intelectuales, saberes y voces sean tratadas de modo igualmente subalternizado, más allá de las condiciones sociales los mantienen en un lugar silenciado estructuralmente (Ribeiro, 2018). Dar lugar en el curso a protagonistas afro para establecer un espacio de pensamiento y reflexión a partir de su experiencia es una forma de trabajar para superar ese lugar silenciado estructuralmente. Abrir un espacio que rompa con concepciones prejuiciosas y que a la vez ayude en la construcción de nuevas formas de relacionamiento, es la aspiración del curso.

El curso fue estructurado en cinco bloques, en la primera edición los contenidos propuestos fueron:

- Unidad 1. Diferenciación, discriminación, exclusión y racismo. Procesos y manifestaciones.

- Unidad 2. «Entramos negros, salimos afrodescendientes». Contexto histórico, político y social en torno al surgimiento del concepto de Afrodescendencia.

- Unidad 3. Bases teóricas de la interseccionalidad.

- Unidad 4. Acciones afirmativas: Reconocimiento, reparación y equidad.

- Unidad 5. La mirada internacionalista.

Durante la segunda edición los contenidos fueron revisados con base en el logro de los objetivos pedagógicos alcanzados en 2019 y por la urgencia coyuntural que imprimió la pandemia, por lo cual los contenidos propuestos fueron:

- Unidad 1. Una mirada interseccional sobre el impacto del covid-19

- «Raza», clase, género. Tríada de base

- Racialización de la pandemia 
- Unidad 2. La mirada desde la acción social solidaria

- Organización y acciones frente a la pandemia desde una mirada antirracista

- Unidad 3. La mirada desde el campo de la salud

- Accesos y resistencias en la salud en relación con la afrodescendencia

- Unidad 4. La mirada internacionalista.

- Impactos de la pandemia en la diáspora afrolatinoamericana

- Unidad 5. Racializada y femenina

- La pandemia en relación con las mujeres afrodescendientes.

Cada estudiante participó del curso en su calidad de egresado, estudiante de grado, profesional o activista militante independientemente de su filiación a una organización. Es decir, las organizaciones estuvieron presentes en forma indirecta a través de la participación de sus miembros. En ambas ediciones se contó con un promedio de veintidós participantes y sus perfiles diversos habilitó debates e intercambios acerca de temáticas amplias tales como identidades étnicas, discriminación, racismo, xenofobia, machismo, distribución de la riqueza, equidad/inequidad, barreras de acceso a los servicios esenciales, políticas públicas, políticas sociales focalizadas, acciones afirmativas, género y masculinidades, masculinidades atravesadas por lo racial, maternidades, paternidades, relaciones sexo afectivas disidentes y birraciales entre otras.

Quedó en evidencia la necesidad de tener a disposición más y mejores materiales que nos acerquen a las temáticas vinculadas a la afrodescendencia, el racismo, sus aspectos históricos, los procesos nacionales e internacionales de abolición de la esclavitud y lucha contra el racismo, pues, incluso participantes profesionales, docentes de educación media o universitaria nos planteaban con asombro no conocer en profundidad estos temas y en la inmensa mayoría de los y las participantes estos cursos los acercó por primera vez a las temáticas desarrolladas.

Por estas notables lagunas de la educación formal uruguaya es que sostenemos con vehemencia, que la afrodescendencia posee un no lugar en el sistema educativo nacional.

Llamamos no lugar a aquellos espacios en los que no hay estricta correspondencia entre la disposición espacial y la disposición social. El antropólogo francés Marc Augé acuñó el término para referirse a los lugares de consumo, comunicación y circulación de la «sobremodernidad», al entender que «si un lugar puede definirse como lugar de identidad, relacional e histórico, un espacio que no puede definirse ni como espacio de identidad, ni como relacional ni como histórico, definirá un no lugar» (Augé,2006, p.83). Al seguir este postulado, sostenemos que la afrodescendencia ha devenido en un no lugar dentro de la institucionalidad educativa y, muy especialmente, en el ámbito universitario. 
En Uruguay, como en el mundo, es evidente que la historiografía; canónica blancaoccidental que no da lugar a una perspectiva afro, al establecer un sujeto normativo que no la contiene, ha dominado el campo de estudios sobre la cuestión afrodescendiente. Por tanto, esto ha producido un correlato a nivel del imaginario académico y social que se visualiza en que los y las afrodescendientes solo somos mencionados y cobramos algún tipo de significación como parte de la historia previa al proceso de gesta del Estado-nación (Martínez et al., 2020, p. 173).

Con esto, evidenciamos como equipo docente la urgente necesidad de que se cumpla lo establecido en la Ley n. ${ }^{\circ}$ 19.122, que en su artículo 8 plantea:

Se considera de interés general que los programas educativos y de formación docente, incorporen el legado de las comunidades afrodescendientes en la historia, su participación y aportes en la conformación de la nación, en sus diversas expresiones culturales (arte, filosofía, religión, saberes, costumbres, tradiciones y valores) así como también sobre su pasado de esclavitud, trata y estigmatización, promoviendo la investigación nacional respectiva (Uruguay, 2013).

Creemos firmemente en que, la incorporación de estas recomendaciones en la educación y el sistema educativo en su conjunto fortalecerá la construcción de ciudadanía de toda la población al tiempo que la calidad democrática de nuestra educación pública, gratuita y universal.

\section{Comentarios finales}

En los intercambios y devoluciones del curso constatamos la necesidad de generar mayor cantidad de cursos de formación a nivel de grado, posgrado y educación permanente, nivelación, actualización y profundización, e incluso de formación de formadores en los cuales sea posible no solo vincularse con referentes académicos, sino también con actores sociales y comunitarios inmersos en las problemáticas abordadas. Esto atiende al primer acuerdo grupal al que pudimos arribar que señala la escasa presencia de la temática afrodescendiente en las líneas de investigación y planes de enseñanza de la Udelar. Hay que señalar si bien existen espacios de formación, investigación y extensión sobre este tema es necesario una mayor visibilidad y coordinación entre ellos de manera de fortalecer y posicionar la temática en la Udelar.

El segundo acuerdo grupal radicó en que, a pesar de la complejidad que la temática reviste no se ve lo suficientemente reflejada en los contenidos curriculares. Esto puede fomentar la ausencia de pensamiento crítico y abonar la idea de que en Uruguay no existen problemas relacionados al racismo, o, que el racismo no está tan presente como en otros países, minimizar sus causas y efectos y, por ende, las experiencias de desigualdad e inequidad de las poblaciones afrouruguayas y afrodiaspóricas en el país.

Un tercer acuerdo unánime consistió en la urgencia de potenciar un diálogo interdisciplinario tanto de los conceptos de raza, racismo, discriminación racial, 
antirracismo, negritud, afrodescendencia y todos sus procesos desde un abordaje que focalice en las relaciones humanas y de cuenta de la complejidad de estos procesos a nivel nacional, para lograr respuestas de acción directa a las manifestaciones concretas con que nos encontramos a diario, desde donde superar la deshonestidad con que se esconde la existencia del racismo en Uruguay y ser capaces así de identificar sus formas de (re)producción.

Confirmamos con pesar, pero henchidos de esperanza a través de estos intercambios, la vigencia de una frase del Manifiesto liminar de la Federación Universitaria de Córdoba (Universidad Nacional de Córdoba, 1918) «los dolores que nos quedan, son las libertades que nos faltan». Caminamos con la convicción de que solo podremos conquistar estas libertades adeudadas fomentando la construcción de espacios formativos que integren al currículum oficial contenidos didácticos y pedagógicos apropiados, materiales que promuevan aquellas historias silenciadas, protagonistas olvidadas y relatos acallados. Espacios formativos que integren estos contenidos en conexión con el devenir social y no dejen el tema restringido al campo historiográfico lejano e inconexo con la actualidad, pues eso fomenta la reproducción de estereotipos, prejuicios y actitudes axiológicas que la ley no puede cambiar. Y son, justamente, las fundamentales de trabajar para dar cambios culturales profundos. Allí reside entonces, el compromiso educativo afrocentrado desde un enfoque de derechos humanos.

\section{Referencias}

AugÉ, M. (2006). Los no lugares, espacios del anonimato. Una antropología de la sobremodernidad. Barcelona: Gedisa.

De Sousa Santos, B. (2009). Una epistemología del sur: la reinvención del conocimiento y la emancipación social. Ciudad de México: Siglo Veintiuno Editores-Clacso.

FANON, F. (2008). Pele negras, máscaras brancas. Salvador: EDUfBa.

GARCía, J. (2010). Afroepistemología y afroepistemetódica. En S. WALKer (Comp.), Conocimiento desde adentro. Los afrosudamericanos hablan de sus pueblos y sus historias (pp. 69-87). La Paz: Fundación Pedro Andavérez Peralta, Afrodiáspora, Fundación Interamericana, Organización Católica Canadiense para el Desarrollo y la Paz, Fundación PIEB.

García, J. (2018). Afroepistemología y pedagogía cimarrona. En R. CAmpoalegra Septien (Coord.), Afrodescendencias. Voces en resistencia (pp. 59-74). Clacso. Recuperado de http://biblioteca.clacso. edu.ar/clacso/se/20180712070816/Afrodescendencias.pdf.

Grosfoguel, R. (2018). ¿Negros marxistas o marxismos negros?: Una mirada descolonial. Tabula Rasa, (28), 367-385.

Instituto Nacional de Estadística (2011). Censo 2011. Recuperado de https://www.ine.gub.uy/web/ guest/censos-2011.

Martínez, L., Olivar, F., Pereira, V., y Pereyra, J. E. (2020). Espacios afrocentrados en el ámbito universitario: recorridos, hallazgos y reflexiones. En M. Olaza (Coord.), Desigualdades persistentes, identidades obstinadas. Los efectos de la racialidad en la población afrouruguaya (pp.167-184). Montevideo: Doble Clic Editoras. 
Martinis, P. (2016). La educación como derecho. En J. G. Lagos (Coord.). 70 años. Facultad de Humanidades y Ciencias de la Educación: 70 años (pp. 18-19). Montevideo: FHCE, Universidad de la República-La Diaria.

MunAnga, K. (2008). Rediscutindo a mestiçagem no Brasil: identidade nacional versus identidade negra. Belo Horizonte: Autêntica.

Munanga, K. (2020, junio 22). Caminhos para uma Educação Antirracista. Parte 1. Recuperado de https:// www.youtube.com/watch?v=fqs_02TgKbk.

Pereyra, J. (2020). Pensar la educación, soñar el futuro. D’Cimarrón, (11). Recuperado de https://dcimarron.org/index.php/columnistas/37-dcimarron-n-11/296-pensar-la-educacion-sonar-el-futuro.

Ribeiro, D. (2018). Breves reflexiones sobre Lugar de Enunciación. Relaciones Internacionales, (39), 13-18. Recuperado de https://revistas.uam.es/relacionesinternacionales/article/view/10o12.

Silva Bento, M. A. (2002). Branqueamento e branquitude no Brasil. En I. Carone y M. A. Silva Bento (Orgs.), Psicologia social do racismo: estudos sobre branquitude e branqueamento no Brasil (pp. 25-28). Petrópolis: Vozes.

Tommasino, H., Cano, A., Castro, D., Santos, C., y Stevenazzi, F. (2017). De la extensión a las prácticas integrales. Recuperado de http://beu.extension.unicen.edu.ar/xmlui/handle/123456789/59.

Universidad Nacional de Córdoba (1918). Manifiesto liminar. Recuperado de https://www.unc.edu.ar/ sobre-la-unc/manifiesto-liminar.

Uruguay (2013, agosto 21). Ley n. ${ }^{\circ}$ 19.122: Fijación de Disposiciones con el Fin de Favorecer la Participación en las Âreas Educativa y Laboral de los Afrodescendientes. Recuperado de https:// www.impo.com.uy/bases/leyes/19122-2013/4. 